\title{
Bio Signal Conditioning and Processing For Biological Real Time Applications Using Mixed Signal Processor
}

\author{
Smt Usha.A ${ }^{1}$, B.Ramachandra ${ }^{2 *}$ and M.S. Dharmaprakash ${ }^{3 *}$
}

${ }^{1}$ Department of E \& E Engg, IEEE Member BMSCE, Bangalore

${ }^{2}$ Department of E\&E Engg, PESCE, Mandya

${ }^{3}$ Department of Chemistry, BMSCE, Bangalore

\begin{abstract}
In medical and health care applications it was always desirable to perform pre-processing and conditioning on the sensor raw output before data acquisition. Based on this requirement, biosensor was proposed and designed using conducting polymers and nano - materials which was having In- situ Signal Conditioning Electronics that eliminates need for additional on board hardware circuitry. This advanced signal-conditioning circuit mainly consists of an ASIC with associated components that process the raw output from bio-sensor. The ASIC works with lowvoltage and low-current, with wide operating temperature range. The bio-sensor, which was designed very much sensitive to room temperature and pressure. Nano-bio sensor ASIC operates at $+5 \mathrm{~V}$ input and provides an output of 0 to $500 \mathrm{mV}$ for absolute sensor output of 0 to 500 nano-amps or pico-amps. Further efforts were initiated to incorporate high resolution $A D C$ with the signal conditioning electronics that gives a serial digital output directly using SPI interface on Mixed-Signal Processor (MSP).

This research work aimed at suitable signal-conditioning and processing electronics using ASIC on MSP. The ASIC designed expected to have various on-chip programmable features for enabling interface compatibility with wide variety of (sample/analyte) sensors and to achieve process variations using mixed signal processor (MSP).

The work is focussed on Optimised Design of Smart Sensor using Conducting Polymer and Gold/Silver/CNT Nanocomposites .Polyaniline (PANY) has been selected as the Conducting polymer on which surface CNT thin layer will be deposited using Sol-Gel technique.Here more emphasis is given to Bio-Signal (which was generated or simulated by the electronic circuit in the laboratory) Conditioning and processing using MSP for biological real time applications.Further the synthesis and characterization of the thin film or the designed sensor will be carried out at IISC Nano-FAB lab under INUP programme.
\end{abstract}

Keywords: Bio-sensor; Signal Conditioning and processing; Mixed signal processor

\section{Introduction}

Most of the smart devices or structures include basically a sensor, signal conditioning circuitry, ADC, switch and a processor. Sensors designed may be of mechanical sensors or actuators, chemical sensors, gas or bio-sensors for telecommunication, food, pharmaceutical, bio-medical areas etc. As application area increase, research has to be focused on new materials for ease of fabrication process. The sensor designed and synthesized is expected to be of high conductivity and high integration compatibility with designed signal-conditioning circuitry and processing which can be easily performed using a simple and cost-effective lower end processor. Many signal- processing applications have some kind of real-time requirements and applications. Hence focus will be on those systems whose response time must be within few milliseconds to a few seconds.

Several Real Time Systems are embedded in sensors and actuators and function as optimized digital controllers (Figure 1).

The term 'plant' refers to a controlled system, for example: an engine, an aircraft, a patient etc. The state of the plant (patient) is sensed and monitored by sensors and can be varied by actuators. The real-time computing system estimates from the sensor readings, current -state of the plant (patient) and computes a control output based on the difference between the current state and the desired state (that is reference input). These digital controllers are cost-effective and widely used.

Important examples of real-time systems include digital control, command and control, optimal control, power control, signal process-

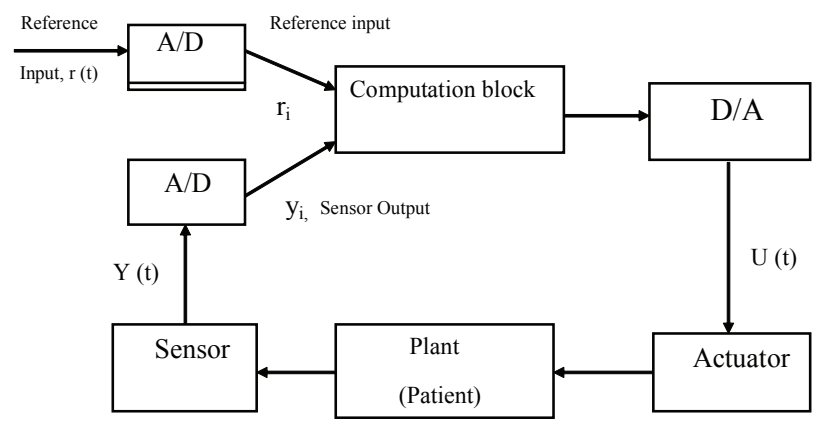

Figure 1: Real Time System.

ing \& telecommunication systems. The complexity of the higher end RealTime Control System arises in addition to its complicated and dedicated specific applications. This often interfaces with the plant or patient and other Signal Conditioning Circuitry, easily and effectively with much greater performance.

*Corresponding author: Usha A Joshi, Department of E \& E Engg, IEEE Member BMSCE, Bangalore, India, E-mail: ushaajoshi@yahoo.com

Accepted June 20, 2011; Published June 29, 2011

Citation: Smt Usha A, Ramachandra B, Dharmaprakash MS (2011) Bio Signal Conditioning and Processing For Biological Real Time Applications Using Mixed Signal Processor. J Biosens Bioelectron 2:105. doi:10.4172/2155-6210.1000105

Copyright: @ 2011 Smt Usha A, et al. This is an open-access article distributed under the terms of the Creative Commons Attribution License, which permits unrestricted use, distribution, and reproduction in any medium, provided the original author and source are credited. 
Citation: Smt Usha A, Ramachandra B, Dharmaprakash MS (2011) Bio Signal Conditioning and Processing For Biological Real Time Applications Using Mixed Signal Processor. J Biosens Bioelectron 2:105. doi:10.4172/2155-6210.1000105

Page 2 of 4

\section{Device design}

Design and development of thin-film sensor is a technique that facilitates a structural designer to achieve the desired characteristics of a Smart-Sensor or Structure. The Optimization procedure is employed for the synthesis of Bio-Sensor for Leukocyte counts in blood sample (Figure 2).

Conducting Polymer, such as Pollypyrrole (PPY) and Polyaniline (PANY) has shown a special capability to rapidly and reversibly switch between different 'Oxidation states', which plays a critical role in BioMedical Real Time Applications. In addition various biomolecules can be incorporated into conducting polymer by Electro-Chemical Polymerization. These two abilities make conducting polymers potential candidates for application in the area of Bio-Sensors. The design and synthesis of sensor thin film using conducting polymers and nanomaterials thus has been recently an increasingly important subject of intensive research.

Designed Bio-Sensor, Polyaniline (PANY) thin film doped with CNT deposited on the ITO Coated Substrate (Sio2) has been applicable for various biological sensors because of its high electrical conductivity in wide range of $\mathrm{Ph}$ values, flexible method of preparation, ease of surface modification, excellent environmental stability, ion-exchange capacity and bio-compatibility.

A suitable oxidizing agent (enzyme or antibody) to increase the conductivity of sensor film is coated on the surface of PANY doped with CNT. Our objective is to produce continuous PANY films with large surface area to ease the immobilization of enzyme and to enhance the concentration of enzyme redox centers.

\section{Signal - Conditioning Circuit}

Signal conditioning means manipulating an analog signal in such a way that it satisfies the requirements of the next stage for further processing. Signal conditioning includes amplification, filtering, con-

\section{Device design :}

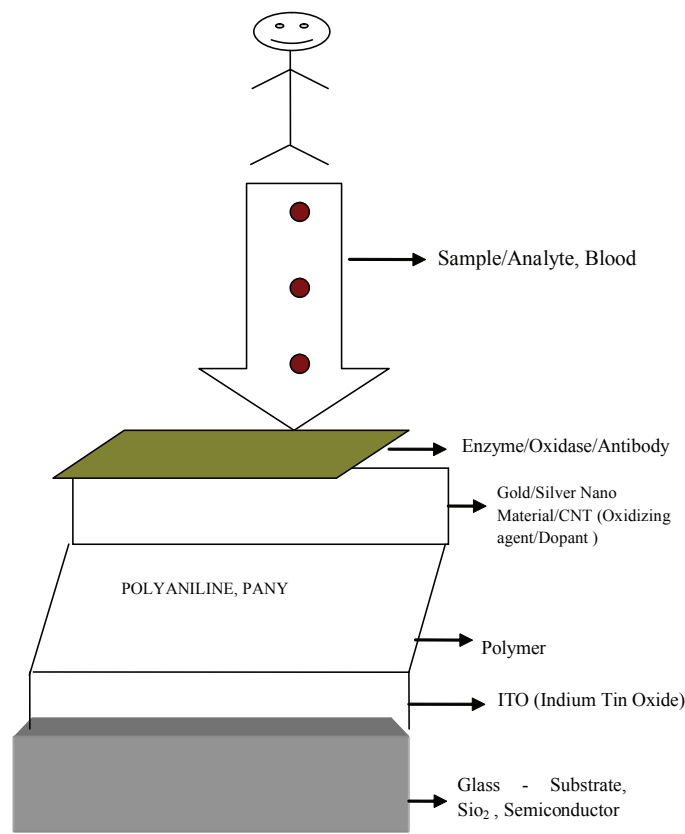

Figure 2: Sensor Thin Film verting, range matching, isolation \& many other processes required to make sensor output suitable for further processing after acquisition, study and analysis.

OP97FP OPAMP is ideal for applications in precision long-term circuits, with very low bias current of 100PA over wide temperature range of $-40^{\circ} \mathrm{c}$ to $85^{\circ} \mathrm{c}$, offset voltage 20 micro volts maximum, input offset current of 750PA and very low-power consumption of $85 \mathrm{mw}$. Output from the Bio-Sensor which is of the order of nano amps is connected to the inverting terminal of the opamp with a feedback resistor $\mathrm{Rf}$ of $5 \mathrm{M}$ ohms, so that an input current of 1 nano-amp will produce an output voltage of 5 milli volts. Hence for an input current signal of ( 0 to 500) nano-amps connected to the opamp OP97FP produces an output

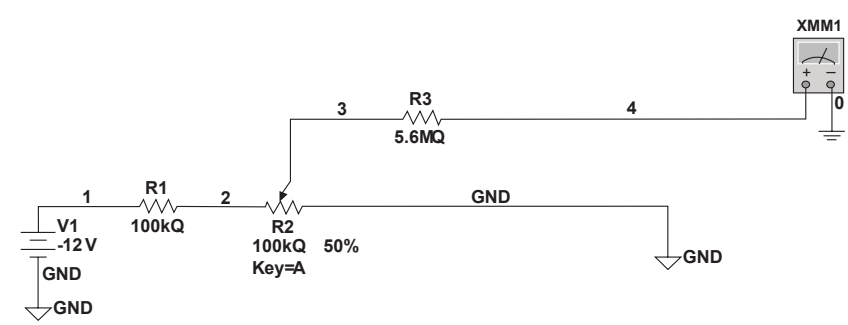

Figure 3: Nano - Source (Bio-Sensor output simulated using above circuit, which produces 0-500 Nano Amps in the laboratory).

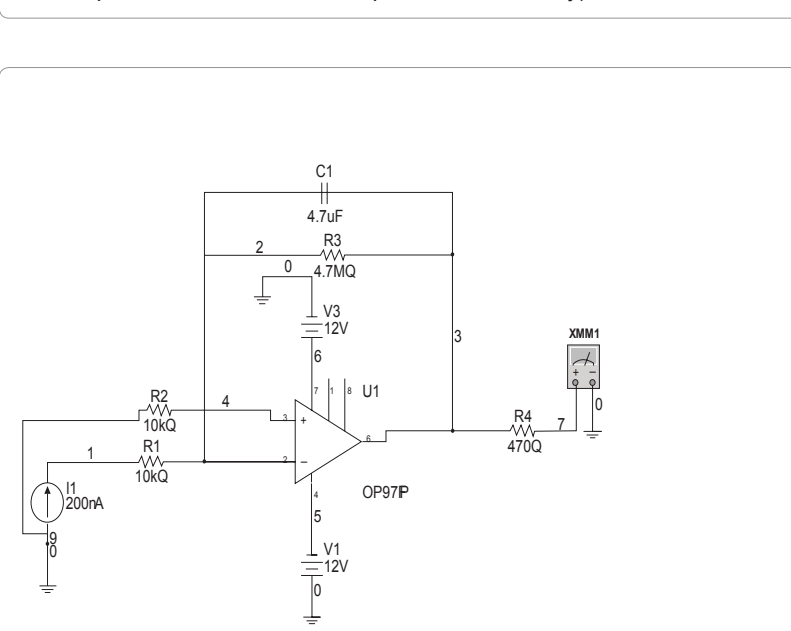

Figure 4: Current to Voltage Converter using OP97FP OPAMP.

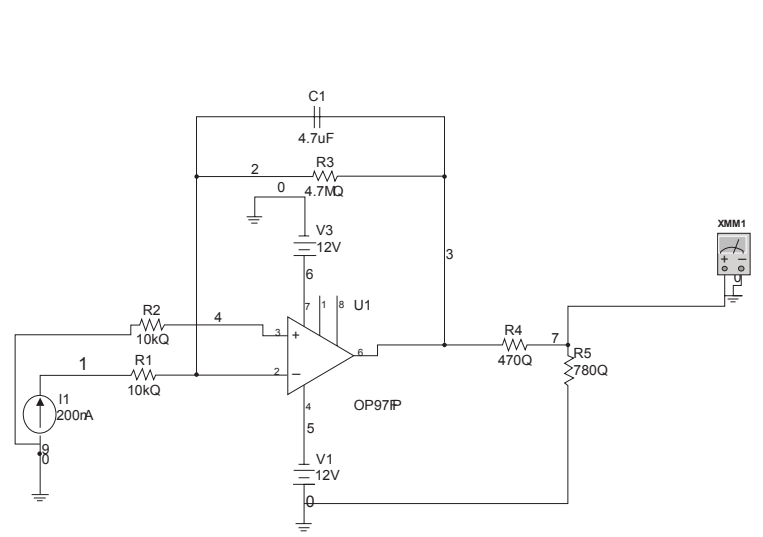

Figure 5: Signal Conditioning Circuit using OP97FP OPAMP. 
voltage of (0 to 500) milli volts which is later amplified to achieve 0 to +5 volts at the output stage (Figures $3,4,5$ ).

\section{Signal Processing Using MSP}

MSP-430 is an Ultra-Low Power 16-Bit Microcontroller family from Texas Instruments. MSP-430 family of Microcontrollers find widespread use in applications such as Energy meters, Medical applications and Wireless Sensor Networks. The combination of the tiny MSP430FG4618 and the highly integrated MSP processor provides nearly various options of peripherals available from the MSP430 family. The integrated TI wireless evaluation module and the large amount of memory RAM on the MSP430 processor makes it an ideal platform for wireless and bio-medical applications. The wide range of that integrated peripherals and hardware connectivity proves MSP430 will be a better choice to develop standalone system especially in medical diagnosis.

MSP430 has the basic facility of low supply voltage range of (1.8V$3.6 \mathrm{~V})$, ultra-low power consumption, 16-bit RISC architecture, 16-bit timers A and B \& with three capture/compare registers, universal serial communication interface, 10-bit analog-to-digital (A/D) converter on-chip with internal reference, $\mathrm{S}$ and $\mathrm{H}$, auto-scan \& Data-Transfer Controller, Two-Configurable opamps, serial on-board programming, on-chip emulation module etc. Because of the above features in this research work MSP430 appears to be the best choice for processing of the conditioned output data obtained from the OP97FP opamp. DC output voltage of the Signal Conditioned circuit (0 to $500 \mathrm{mV}$ ) in turn is applied to the MSP430 chip for further processing that is filtering of low frequency signals or noise present at the output of bio-sensor. Later by executing a suitable code in Embedded - C, application software program can be implemented on MSP processer to get the required display on LED/LCD as the Leukocyte count varies in blood sample/ analyte (Figure 6).

\section{Results and Discussion}

For bio-sensing, labeling is a basic requirement for detecting and analyzing biomolecules \& bio-reactions. Labeling is however an expensive and time consuming process. The ability of label free detection, scalability to allow massive parallelization and sensitivity of the detection range are the important requirements for a future generation of bio-sensors. Therefore, researchers are trying to develop label free biosensing, non-invasive or minimum invasive techniques. For label free

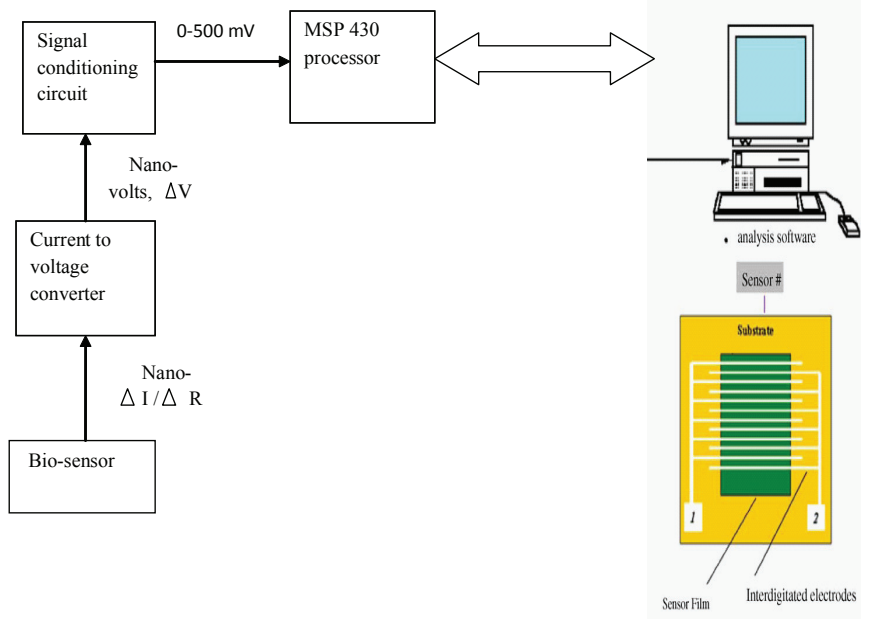

Figure 6: Signal Processing Circuit. detection, currently there are three popular candidates: Surface Plasmon resonance (SPR), optical method design (OMD) and Paramagnetic based (PMG) bio sensors. SPR can analyze only one sample at a time and it is not economical also. Moreover, detection of small molecules is it's another limitation. Although more sensitive than SPR, PMG bio- sensors has limited scalability because of its construction, usability which depends strongly on sensor surface. So the electrochemical based bio-sensor appears to be the right choice for the optimized design of bio-sensor for Leukocyte counts by minimum-invasive technique.

Bio-Signal is acquired or captured or trapped at the output of BioSensor which is of the order of nano-amps or sometimes it may be of the order of Pico-amps. Using a suitable Current to Voltage Converter, (Analog Devices family chip) nano-bio current signal is converted into nano-voltage range. Then the signal voltage is passed through an amplifier stage which results in modulating the output in the order of millivolts. By designing a specific Signal Conditioning Circuit, the milli-volt signal is converted into variable voltage ( 0 to +5 volts) range, which later on digitized by implementing Unipolar ADC Chip.

The ASIC Chip designed enable output adjustment, with minimum noise and offset voltage signal conditioning, amplification and processing the acquired signal of the Bio-sensor. ASIC operates at $+5 \mathrm{~V}$ input and provides an output of 0.1 to $2.0 \mathrm{~V}$ for an input current signal ranging from 0 to 500 nano-amperes, released by the bio-sensor as leukocyte count varies in the blood sample. Presently efforts are initiated to incorporate high resolution and precision 10-bit ADC with the MSP430 Processor electronics that produces a serial digital output display of information about various patients located at remote or distant places.

This research work, is focused on design of Bio-Sensor, acquiring signal data of the sensor i.e. by constructing a nano-current-source circuit (0-500) nano-amps whose output was proportional to the designed bio-sensor signal output and later building a suitable Signal Conditioning Circuitry using (Analog Devices - Chip). Conditioned signal was further processed by employing MSP-430 Processor.

The inaccuracies accumulated under unknown environmental or abnormal conditions are found to be invalid and ignored where other characteristics such as cost, time and reliability are more important. Some of the new potential - methods are suggested in future work for significant reduction of the inaccuracies. A comparative study and analysis has been carried out when the signal conditioned output is interfaced with Mixed Signal Processor (MSP-430) for further processing and a suitable algorithm can be implemented for displaying of the captured and acquired signal data from the sensor. The Prototype Model developed in this work will be useful for Structural Health Monitoring Applications.

In the (Electrical and Electronics) laboratory Bio-Sensor output had been simulated by designing and constructing a typical Nano-Source Electronic circuitry which is capable of producing practically (0 to 1000 nano amperes) current signal. Later the output of the Nano-Source circuit was conditioned and converted efficiently into milli-volts( 0 to 1000 $\mathrm{mV}$ ) signal using OP97FP OPAMP. Further this signal was amplified efficiently into $(0$ to +2$)$ volts which were processed by employing a simple and cost-effective MSP-430 Processor.

In this research work we have processed the Bio-Signal which is of the order of ( 0 to $500 \mathrm{mV}$ ) generated or acquired from the simulated Bio-Sensor (Nano-Current Source) circuit efficiently using a typical Mixed-Signal Processor. Real-Time Bio-Signal since are usually very weak in nature and will be comprised of interferences and noises, it was required to filter the unwanted components from the acquired Bio- 
Citation: Smt Usha A, Ramachandra B, Dharmaprakash MS (2011) Bio Signal Conditioning and Processing For Biological Real Time Applications Using Mixed Signal Processor. J Biosens Bioelectron 2:105. doi:10.4172/2155-6210.1000105

Signal using MSP-430 Processor and processed signal data or information was displayed on a LCD by writing Embedded "C" Programme.

In future, the Pico-amps current signal output of the BIO-SENSOR can also be acquired, conditioned, processed and analyzed using Data Acquisition Card or DMM-Card. Further, based on this information or data medical supervisor can subscribe the required specific suggestions or treatment to the people who are in need in this society.

The feasibility of using the inherently Smart Structures integrated with ASIC results for low-cost, robust smart sensor for health monitoring has been investigated. Specific needs are identified in the SensorFilm Design Technology and design of suitable Signal- Conditioning Circuit for nano-bio-current signal output of the sensor. For this OP97FP opamp may be effectively designed along with few passive elements or components. The response of the sensor will be simulated and studied for various samples or analyte at room temperature and pressure (Tables 1, 2, 3).

\section{Conclusion}

In this Research work, PANY Film doped with CNT on ITO coated substrate by Electro-Chemical Method is designed and analyzed. Ultimate objective is to produce signal conditioned output of the Bio-Sensor (simulated output) which is proportional to the Leukocyte counts variations in blood sample. Further conditioned Sensor-Current Signal output interfaced with the Mixed-Signal Processor, i.e. MSP-430, to achieve filtering and displaying of relevant information or message which is cost effective, efficient, programmable and easily compatible with external real world. Relevant Algorithms have been implemented

\begin{tabular}{|l|l|l|l|}
\hline $\begin{array}{l}\text { Bio- signal (Nano - } \\
\text { source output in Nano- } \\
\text { amps) }\end{array}$ & I to V converter & $\begin{array}{l}\text { Amplifier output } \\
\text { (Milli - volts) }\end{array}$ & $\begin{array}{l}\text { Signal condi- } \\
\text { tioned Output } \\
\text { (Volts) }\end{array}$ \\
\hline 0 & 0 & 0 & 0 \\
\hline 100 & 100 & 100 & +1 \\
\hline 200 & 200 & 200 & +2 \\
\hline 300 & 300 & 300 & +3 \\
\hline 400 & 400 & 400 & +4 \\
\hline 500 & 500 & 500 & +5 \\
\hline
\end{tabular}

Table 1: Signal Conditioned Output Table.

\begin{tabular}{|l|l|l|l|l|}
\hline $\begin{array}{l}\text { Bio- signal (nano- } \\
\text { source output in nano- } \\
\text { amps) }\end{array}$ & $\begin{array}{l}\text { I to V con- } \\
\text { verter } \\
\text { (nano- volts) }\end{array}$ & $\begin{array}{l}\text { Amplifier } \\
\text { output } \\
\text { (milli- volts) }\end{array}$ & $\begin{array}{l}\text { Signal condi- } \\
\text { tioned Output } \\
\text { (volts) }\end{array}$ & $\begin{array}{l}\text { A/D } \\
\text { Converter } \\
\text { (binary) }\end{array}$ \\
\hline 0 & 0 & 0 & 0.0 & 00000000 \\
\hline 100 & 100 & 100 & 0.4 & 00110011 \\
\hline 200 & 200 & 200 & 0.8 & 01100110 \\
\hline 300 & 300 & 300 & 1.2 & 10011001 \\
\hline 400 & 400 & 400 & 1.6 & 11001100 \\
\hline 500 & 500 & 500 & 2.0 & 11111111 \\
\hline
\end{tabular}

Table 2: Signal Conditioned and ADC Output Table.

\begin{tabular}{|l|l|l|}
\hline $\begin{array}{l}\text { Bio-sensor output (Simu- } \\
\text { lated using Nano-Source, } \\
\text { in Nano-Amps) }\end{array}$ & $\begin{array}{l}\text { OPAMP,OP97FP } \\
\text { Output, (Milli-volts) }\end{array}$ & $\begin{array}{l}\text { LCD display, } \\
\text { On MSP-430 Processor }\end{array}$ \\
\hline 0 & 0 & Reset status \\
\hline 100 & 100 & WBC count is 10000 , normal \\
\hline 200 & 200 & WBC count is 20000, normal \\
\hline 300 & 300 & WBC count is 30000 , normal \\
\hline 400 & 400 & WBC count is 40000 , abnormal \\
\hline 500 & 500 & Critical condition \\
\hline
\end{tabular}

Table 3: LCD Display of Data Output of Sensor on MSP Processor. on MSP-430 Platform for designing a Standalone Diagnostic System to assist Cancer Patients in the Society.

\section{Acknowledgement}

We acknowledge Chetan Ghatage, M.Tech Student for the support in implementation of Nano-Source and Nano-Bio-Signal Conditioning and Processing Circuit. We also thank Dr Ravishankar Deekshit for extending the Electrical and Electronics Lab Facility while carrying out Research work. Further, we would like to extend our heartfelt thanks to Dr Mahesh for his technical guidelines.

\section{References}

1. Ben Hur V Berges and Luiz Eugenio M de Barros Jr (1999) Design of optical Bio-sensor using polymeric waveguide. SBMO or IEEE MTT-SIMOC 99 Proceedings.

2. Holmes D, Taosun Morgan H, Holloway J,Cakebread J, Davies D (2007) Labe Free differential Leukocyte counts using a Microfabric single cell Impedance Spectrometer. Sensors, IEEE.

3. Lee M, Fanchet PM (2006) Label-Free optical Bio-Sensor using silicon two dimensional photonic crystal. The Insti tute of Optics IEEE.

4. Chang DA, Yen BK Gale (2005) Design \& Fabrication of Multianalyte - capable optical bio-sensor using multi physics approach. IEEE.

5. Mohd Zahid Ansari, Chongducho (2008) Design and analysis of a high sensitive Multianalyte Biosensor for biomedical applications. IEEE.

6. Ohkawa M, Izutsu M, Sueta T (1989) Integrated optic accelerometer employing a cantilever on a silicon substrate. Jap J Appl Phys 28: 287-288.

7. Yunan Cheng, Gangwu, Gustaaf Borghs, MangWang, Hong-Zheng Chen (2008) Electro Chemical Polyaniline/ Polypyrrole Composite Film with Nove Nano-structure and high Bio-Sensitivity. Dept. of Polymer Science and Engineering, State Key Lab of Silicon Materials, China.

8. Michel Bozlan, Fabien Miomande, Jinbo Bai (September 2008) Electrochemical Synthesis and Characterization of Carbon Nano tube or Modified Polypyrrole Hybrids Using a Cavity Micro Electrode. France, Science Direct Journal.

9. Godovsky DY (2000) Device Applications of Polymer Nano Composites. Energy \& Semiconductor Dept, University of Oldenburg, Germany, Advances in Polymer Science 153 Springer-Venlag Berlin.

10. Ananth Suresh, Bhat KN, Vinoy KJ (2009) Smart Sensors and Nanotechnology. IISC, Bangalore.

11. Hua Shao, Dhiraj Kumar,student member IEEE and Kevin L Lear, Member, IEEE, (December 2006)Single-Cell Detection Using Optofluidic Intracavity Spectroscopy. IEEE Sensor Journal, 6.

12. Michael Bozlar, Fabien Miomandre, Jinbo Bai (16 September 2008) Electrochemical synthesis and characterization of carbon nanotube/modified polypyrrole hybrids using a cavity microelectrode. Elsevier, France. 